\title{
Geothermal Power: Monitoring the Building Response During Installation
}

\author{
FABIO CASCIATI, SARA CASCIATI, \\ ALESSANDRO COLNAGHI and LUCIA FARAVELLI
}

\begin{abstract}
The European project G E O F I T (Deployment of novel GEOthermal systems, technologies and tools for energy efficient building retrofitting) is gathering more than 20 partners from all around Europe. Its main objective is to deploy and to integrate advanced methods of worksite inspection, ground research, and building structural monitoring, drilling and worksite characterization into advanced geothermal based retrofitting methods.

This contribution reports the experimental results to be achieved within GEOFIT at the location of specific case studies. In particular standard accelerometric measurements will be collected and compared with the information collected by the geo-radar system made available by one of the partners. This paper focuses on the structural monitoring of a two-story masonry building. The results of a preliminary data collection in the absence of drilling are also reported.
\end{abstract}

\section{INTRODUCTION}

The current European Union (EU) policy aims to increase the amount of green contribution to the energy consumption within the more than 20 European Countries forming the Union. A well promising approach relies on geothermal energy exploitation. The current implementations are still far from urban nuclei, but mainly apply to isolated buildings. Nevertheless, the installation requires to drill under the building and this is a source of vibrations that can result quite dangerous for the target building.

The project G E O F I T [1] planned to implement structural monitoring in some pilot locations. The task is to perform subsequent experimental campaigns to collect information about changes in the structural behavior due to the drilling, regarded as a traumatic event.

Fabio Casciati, DICAR, University of Pavia, via Ferrata 3, 27100 Pavia, Italy, fabio@dipmec.it

Sara Casciati, SIART srl, via dei Mille 73, Pavia, Italy, saracasciati@msn.com Alessandro Colnaghi, SIART srl, via dei Mille 73, 27100 Pavia, info@siart-srl.com Lucia Faravelli, DICAR, University of Pavia, via Ferrata 3, 27100, Pavia, Italy, lucia@dipmec.it 


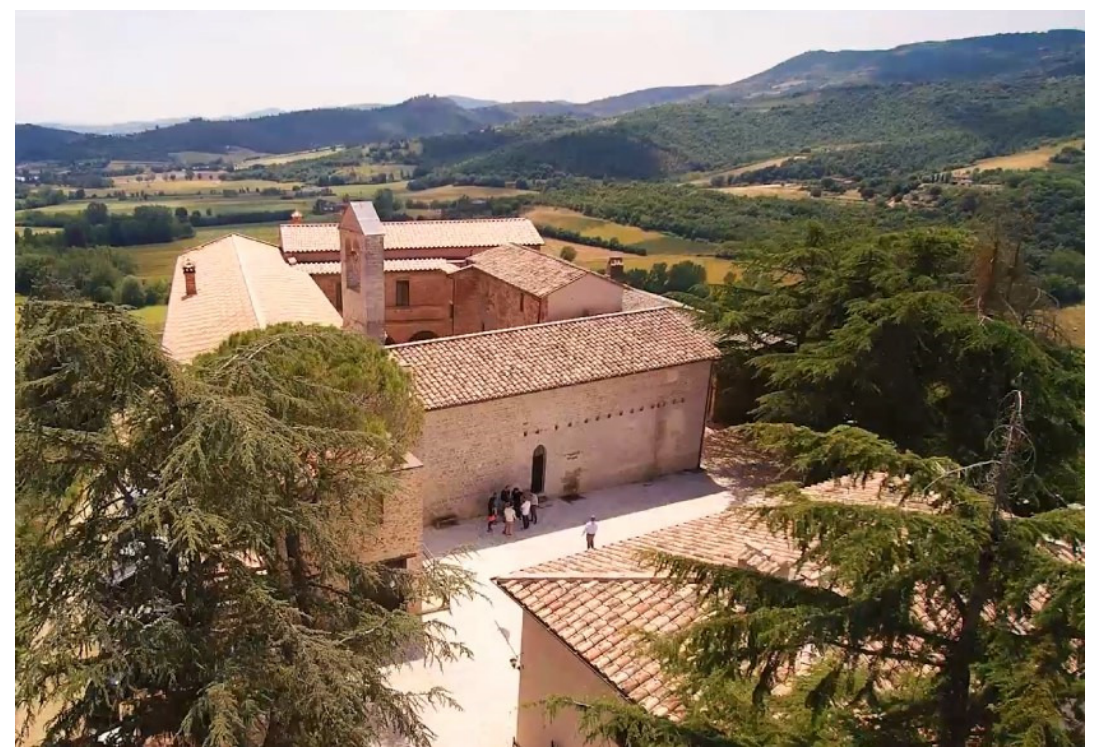

Figure 1. Pilot site at Sant'Apollinare, Perugia.

In particular, the survey is organized in three data campaigns. The preliminary one aims at determining the present state of the structure. During the drilling phase under the building, as required by the installation of the geothermal energy system, a second testing campaign is scheduled. Eventually, a post-drilling phase is supposed to highlight any behavioral anomaly with respect to the building initial state.

One of the pilot locations, consisting of a historical building in masonry (Figure 1) is herein selected as an explicative example. Each campaign will convey four different classes of sensor devices: standard accelerometers, geo-radar, vision-based technology and UAV (unmanned aerial vehicle). The last three items are of the noncontact category, which is regarded as a wished feature. Standard accelerometers are deployed to catch the high frequency vibrations and for sake of comparison.

\section{MONITORING INSTRUMENTATION}

\section{Accelerometers}

The standard monitoring system of tri-axial accelerometers is first discussed. The adopted device is the old, but robust, EPISENSOR Model FBA ES-T with analog output [2]. The advantages of using these sensors are:

- Very-low self-noise, resulting in $155 \mathrm{~dB}$ dynamic range;

- User-selectable full g-scale range: from $\pm 0.25 \mathrm{~g}$ to $\pm 4 \mathrm{~g}$;

- User-selectable full output range: from $\pm 2.5 \mathrm{~V}$ to $\pm 20 \mathrm{~V}$, differential;

- Wide frequency response: from DC to $200 \mathrm{~Hz}$. 
To accurately record accelerations greater than $0.1 \mathrm{~g}$, it is essential, the EpiSensor has to be anchored to the structure. Instead, for weak motion one just places the sensors on the ground, because the weight of the instrument and the friction between the device and the floor, ensure an accurate reproduction of ground motion.

The selected kind of accelerometer is compatible with the wireless acquisition system designed in [3-4] and implemented into several testing applications (see [5-6], among others).

\section{Georadar}

HYDRA-G (Figure 2) is a compact, remote sensing monitoring system designed for early warning and real-time measurements of sub-millimetric displacements in buildings, dams, tunnels, mining infrastructures, and cut-slopes [7].

With a scan range up to 800 meters, the system relies on high-accuracy and resolution radar technology. The acquisition system of HYDRA-G exploits the cutting-edge ArcSAR technology, providing a spatial resolution of centimeters and updated displacement information every 30 seconds.

An optical and infrared HD camera provides real-time visual inspection of monitored areas, and radar data are draped on a 3D model of the scene created by the built-in laser. For post-processing, the HYDRA Guardian software provides an easy-to-use and powerful tool to visualize and interpret radar data, and perform analysis of displacement trends of structure and cut-slope movements. Moreover, SurfScan is a dedicated, real time building displacement and deformation analysis software.

The main benefits of this technology are listed below:

- Non-intrusive technology: no pointers or devices to be installed on the target

- Hyper spatial resolution: to detect even the smallest displacement with submillimetric displacement accuracy

- Fast acquisition rate: 30 seconds for a full resolution scan

- Short range and large angular coverage capability: to track in real-time thousands of points in the monitored scenario

- On-site results: data provided in real time with instant processing

- 3D representation and visual imaging: to ease data interpretation of the monitored area

- Compact and portable solution: easily transportable from a location to another and installed by one single person

- High availability with low maintenance costs: minimal moving parts and low profile design to guarantee robustness and maximum availability in harsh environmental conditions. 


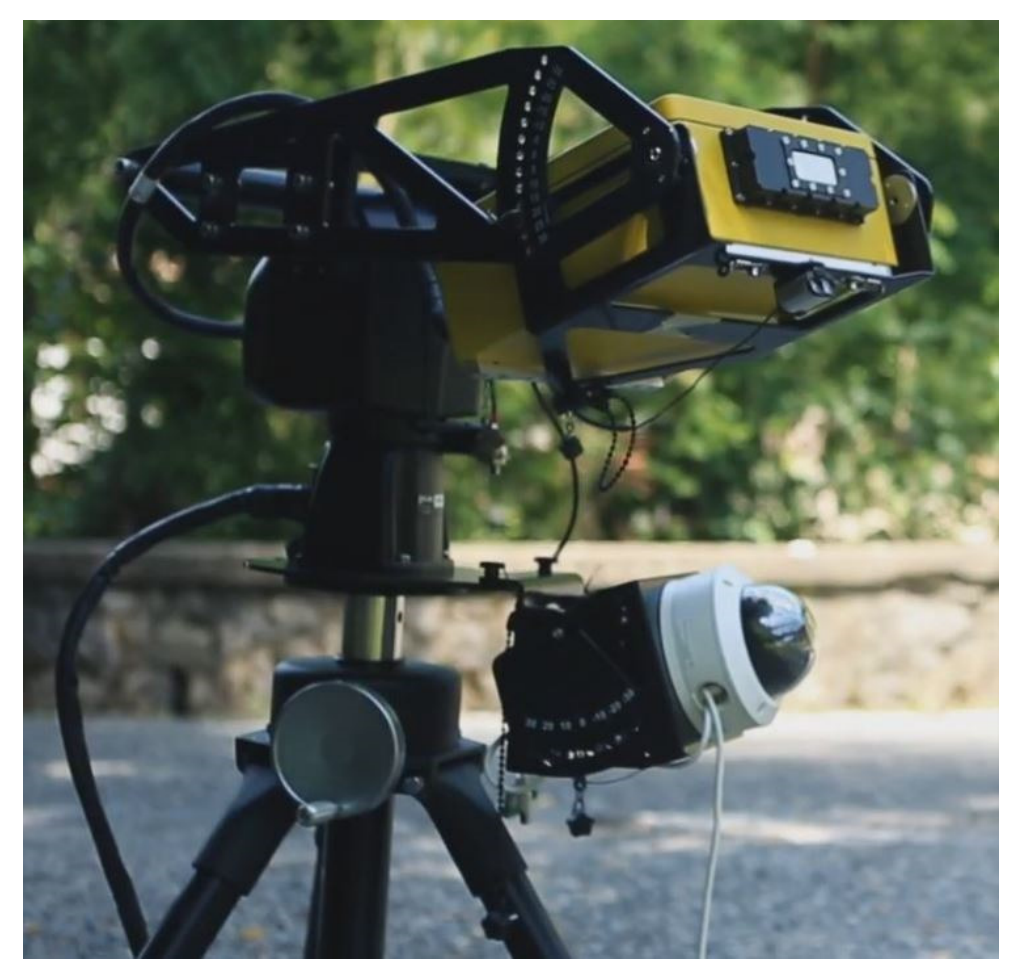

Figure 2. HYDRA-G IDSGeoRadar [8].

\section{Vision - Based - Technology}

In a recent thesis defended at the University of Pavia [9], the author outlines how the use of a smartphone to collect images of a vibrating object is suitable in order to describe the motion of target points (markers). This allows one to detect the frequency content up to $5 \mathrm{~Hz}$. This is not bad for displacements, since the spectrum of such a physical variable decays quickly with the frequency. Of course, the processing requires the adoption of a commercial code as IPWIN60 or to program a specific software within Matlab.

It is worth noticing that the image coming from the smartphone is quite rich (and requires a large amount of storage space). This is why the first step following the acquisition consists in drastically reducing the information content to make manageable the extraction process. Figure 3 provides a short synthesis of the tracking.

\section{UAV (Unmanned Aerial Vehicle)}

Figure 4 shows a non-autonomous unmanned aerial vehicle (UAV), i.e., a drone. Today, UAVs have become a very important resource for surveys and structural and environmental monitoring. The drone allows one to detect and to catalog large areas of the territory, saving time compared to using traditional methods. Today thanks to drones, it is possible to reach narrow places that are difficult to reach.

The drones can be suitably set up with different types of cameras, each with particular characteristics and capable of making these small and versatile aircraft valuable allies in the most diverse fields. 


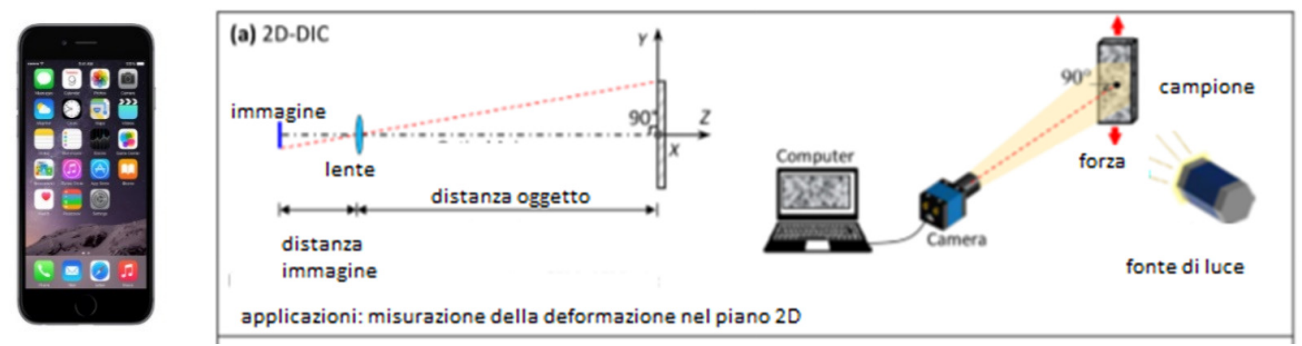

a)
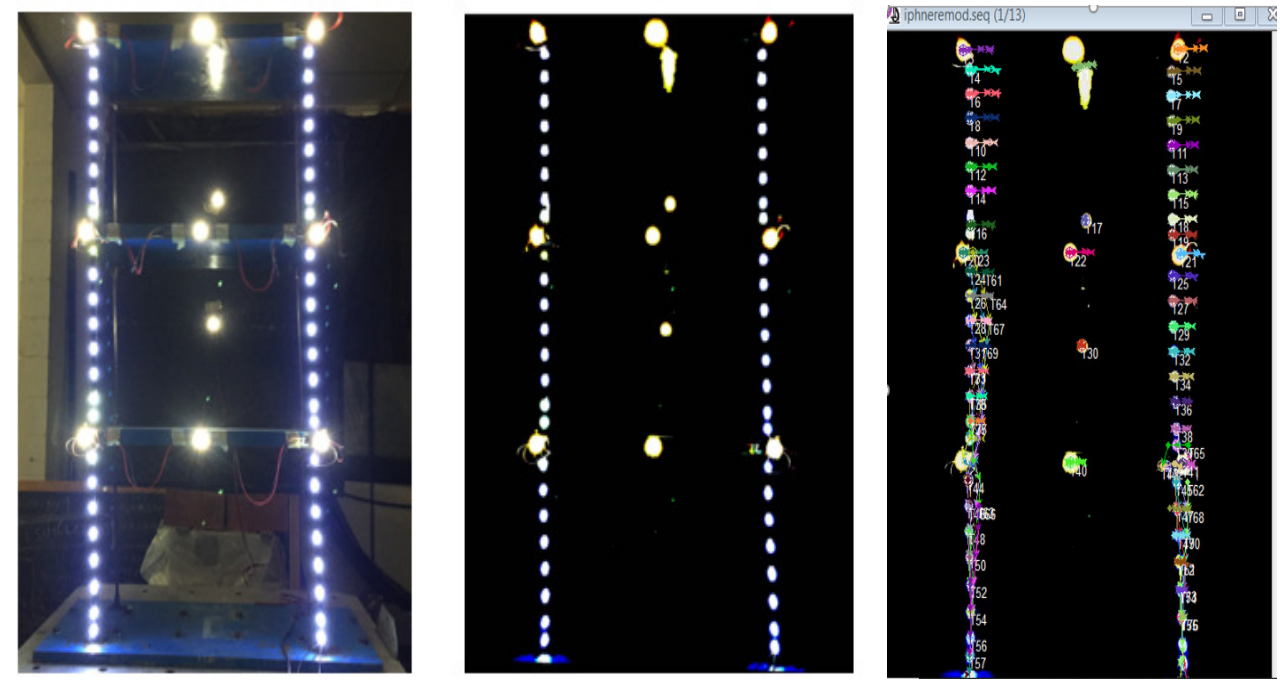

b)

Figure 3. Synthesis of the vision-based approach (from [9]): a) device and procedure; b) collected image, processed image, identification of the markers.

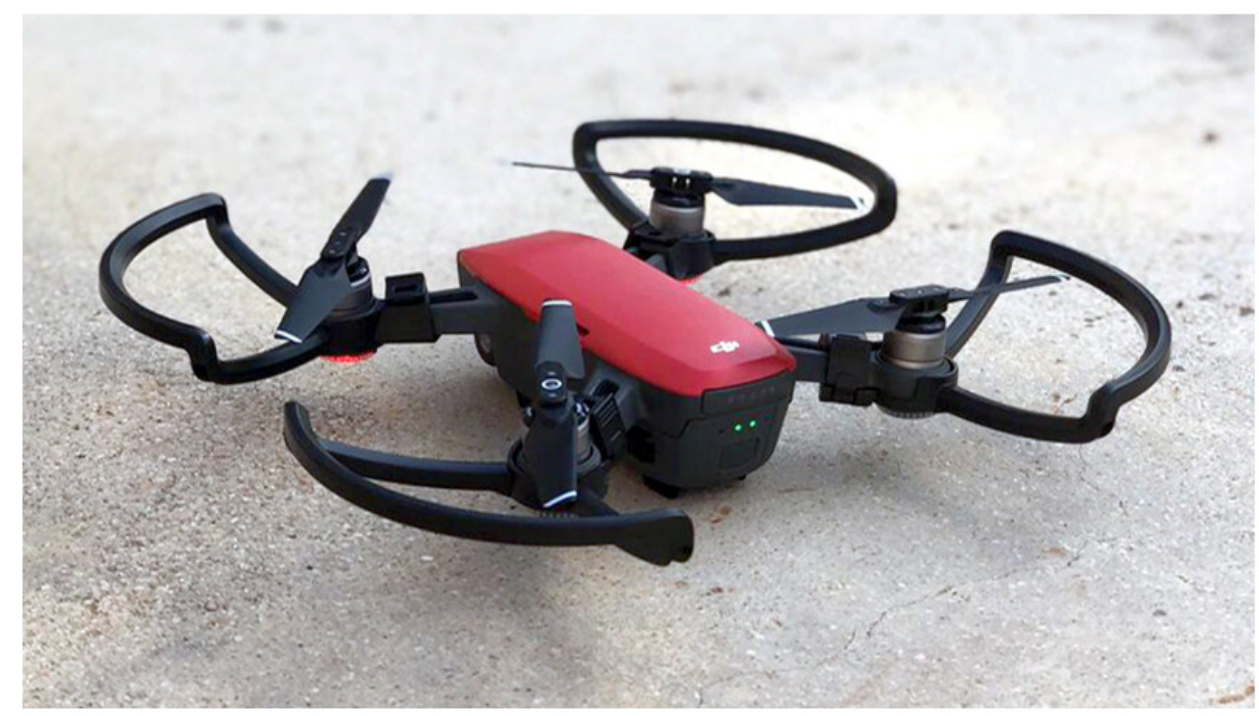

Figure 4. Drone [10]. 
One of the areas in which the application of drones has found fertile ground is thermography. This technique, with the use of special cameras that work in the field of the infrared spectrum, it is possible to detect the different thermal anomalies that are present within the different structures being examined. Thermography has been used for years in various sectors to identify problems related to heat dispersion, liquids or damage. Thermal imaging cameras allow you to create heat maps that indicate the different thermal variations and that, on closer examination, allow one the identification of potentially serious problems. For this reason, one of the main areas in which drone thermography is widely applied is the civil sector and in particular the structures, such as bridges and dams. It is in fact evident the possibility of obtaining images (from the simple click, to the thermal camera analysis, etc.) in parts that can be easily reached by means of the drone, which otherwise would require solutions that are economically more expensive and humanly more risky.

Structural analysis with drones is a fairly recent practice in the engineering sector. The possibilities offered by these unmanned vehicles are manifold and allow to carry out analyzes and verifications that otherwise would require far more expensive and complex equipment.

The use of drones for monitoring brings some significant advantages including:

- Maximum safety for the staff

- You can operate remotely without being in contact with any traffic

- Extensive documentation

- Possibility to archive the results and make the analysis repeatable after months

The same philosophy can be applied for the monitoring of other engineering works. In any case the main advantages are a lower use of resources and time as well as an increase in the level of precision.

Finally, a potential of drones that interests both architects and engineers is that of being able to capture such data sets as to build high-precision 3D models of buildings and structures (Figure 5).
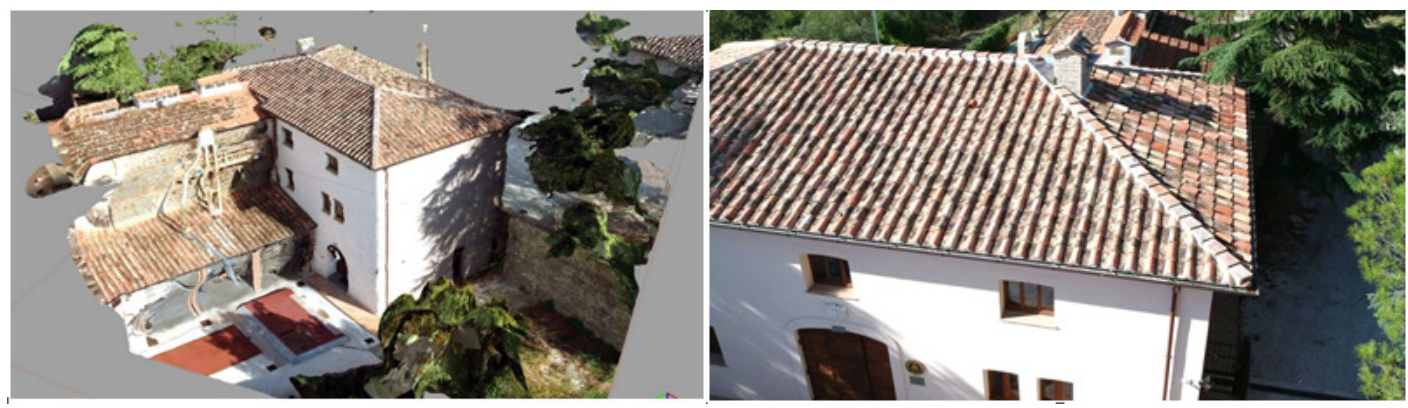

Figure 5. 3D model performed by drone [10]. 


\section{PRELIMINARY ELABORATION FROM THE FIRST TESTING CAMPAIGN}

Traditional electro-mechanical vibrodynes (Figure 6) are excitation equipment capable of delivering rigorously sinusoidal unidirectional dynamic forces. Such equipment is normally made in a single casing (frame) inside which two counterrotating parts are keyed together. The rotary motion of the electric drive motor is programmable and features continuously adjustable rotation speed. Toothed belts to a rotating part and to a counter-rotating part transmit it.

When dealing with historical masonry buildings, the owner is simply afraid for potential damages induced by the inferred vibration. In the case study reported in next section, an agricultural machine working nearby was playing the role of a vibrodyne. It was producing signals showing a suitable frequency content at 0.9 and $1.4 \mathrm{~Hz}$.

The first testing campaign was carried out the first days of May 2019. The wireless accelerometers were placed at three different levels: underground (Work Station 2, WS 2), at the ground floor (WS4) and at the first floor (WS3). (Figure 7) shows the periodogram of the signals recorded in the direction transversal to the masonry wall. The peaks associated with the agricultural machine induced vibration are well outlined, together with the induced vibration at the different levels.

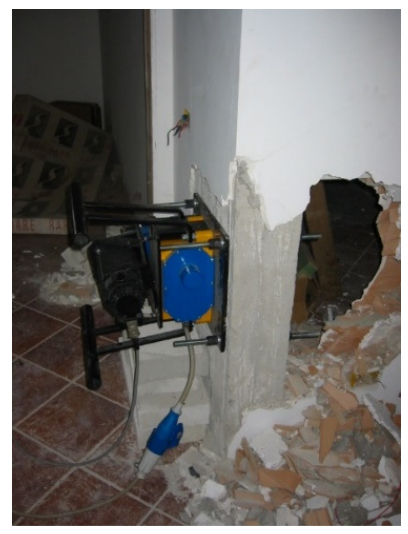

Figure 6. A vibrodyne on a Shear Wall.

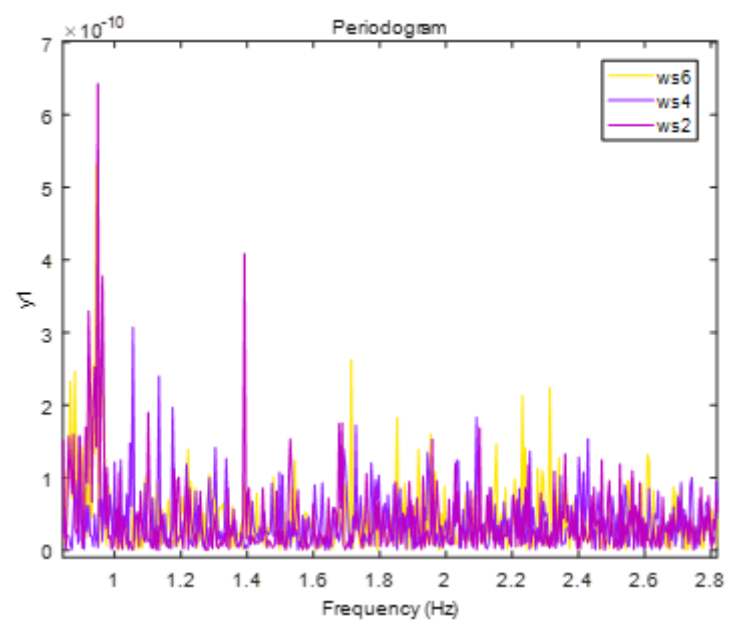

Figure 7. Periodogram from the first test campaign. 


\section{CONCLUSIONS}

In most cases, the monitoring process is limited to the identification of the fundamental modes of vibration and to the evaluation of the amplitude of the responses of the structure as a whole. In the presence of non-symmetrical buildings, or subjected to non-symmetrical loads, measurement positions must be provided to detect torsional vibrations of the building itself. Being well conscious of this situation, the GEOFIT project actors designed the test campaign by exploiting different sensor technologies in order to cover all the aspects that it is likely will be interested in the drilling phase.

\section{ACKNOWLEDGEMENT}

The activity reported in this paper has received funding from the European Union Horizon 2020 research and innovation program under grant agreement No 792210 (Geofit).

\section{REFERENCES}

1. Geofit. "https://geofit-project.eu/"

2. Kinemetrics. "https://kinemetrics.com/post_products/episensor-es-t/"

3. Casciati, S., Z. Chen. 2011. "A multi-channel wireless connection system for structural health monitoring applications" Structural Control \& Health Monitoring, Volume: 18 Issue: 5 Pages: 588-600.

4. Casciati, S., L. Faravelli, Z. Chen. 2012. "Energy harvesting and power management of wireless sensors for structural control applications in civil engineering" Smart Structures and Systems, Volume: 10 Issue: 3 Pages: 299-312.

5. Chen, Z., S. Casciati, L. Faravelli. 2015. "In-Situ Validation of a Wireless Data Acquisition System by Monitoring a Pedestrian Bridge" Advances in Structural Engineering, Volume: 18 Issue: 1 Pages: 97-106.

6. Casciati, F., S. Casciati, M. Vece. 2018. "Validation range for KF data fusion devices" Acta Mechanica, Volume: 229 Issue: 2 Special Issue: SI Pages: 707-717

7. M. Cecchetti, M. Rossi, F.Coppi F. 2018 "Performance evaluation of a new MMW Arc SAR system for underground deformation monitoring", Proceedings Volume 10788, Active and Passive Microwave Remote Sensing for Environmental Monitoring II; 107880B https://doi.org/10.1117/12.2323828: SPIE Remote Sensing, Berlin, Germany

8. IDSGeoRadar. "https://idsgeoradar.com/products/interferometric-radar/hydra-g"

9. Rosadini, L. 2019. "Digital Image Correlation (DIC) come base per il monitoraggio strutturale vision based" (in Italian), Civil Engineering Master Thesis, University of Pavia.

10. R2Msolutions. "http://www.r2msolution.com/engineering-it/drones/" 